\title{
Optimizing Specimen Thickness by Measuring Stain Density in STEM Tomography of Cells and Tissues
}

Qianping He, Guofeng Zhang and Richard Leapman

National Institute of Biomedical Imaging and Bioengineering, National Institutes of Health, Bethesda, Maryland, United States

Stain density is an important parameter for optimizing the quality of ultrastructural data obtained from several types of 3D electron microscopy techniques, including serial block-face electron microscopy (SBEM), focused ion beam scanning electron microscopy (FIB-SEM) and axial bright-field STEM tomography. A simple expression is derived to determine the stain density based on straightforward measurements in the TEM [1]. Numbers of stain atoms per unit volume are determined from the measured ratio of the bright-field intensities from regions of the specimen that contain both pure embedding material and the embedded biological structures of interest. The determination only requires knowledge of the section thickness, which can either be estimated from the microtome setting, or from low-dose electron tomography, and the elastic scattering cross section for the heavy atoms used to stain the specimen.

The measured stain density is then used as a criterion to determine the practical thickness range for performing bright-field STEM tomography at a beam energy of $300 \mathrm{kV}$. We have previously shown that bright-field STEM tomography gives higher spatial resolution than dark-field STEM tomography for thick specimens [2, 3]. In bright field STEM tomography, due to elastic scattering, the usable thickness range of specimen depends on the numbers of heavy atoms ( $\mathrm{Os}, \mathrm{U}, \mathrm{Pb}$, etc.). Our study shows that for the retinal pigment epithelium sample, while the $500 \mathrm{~nm}$ section gives high quality images (Fig. 1A), the image quality deteriorates when sections are $\sim 750 \mathrm{~nm}$ thick (Fig.1B). This is consistent with a measured value of 1.06 atoms $\mathrm{nm}^{-3}$. However, in the section of blood platelets in Fig. 1C, it is possible to reconstruct specimens of thickness $\sim 1.4 \mu \mathrm{m}$, which is consistent with a measured stain density of 0.71 atoms $^{-3}$.

Our results show that, whilst bright-field STEM tomography of thick sections is a powerful technique, it is critical to control the level of heavy atoms that are absorbed during specimen preparation. In this regard, the proposed stain measurement method has proven to be simple and straightforward [4].
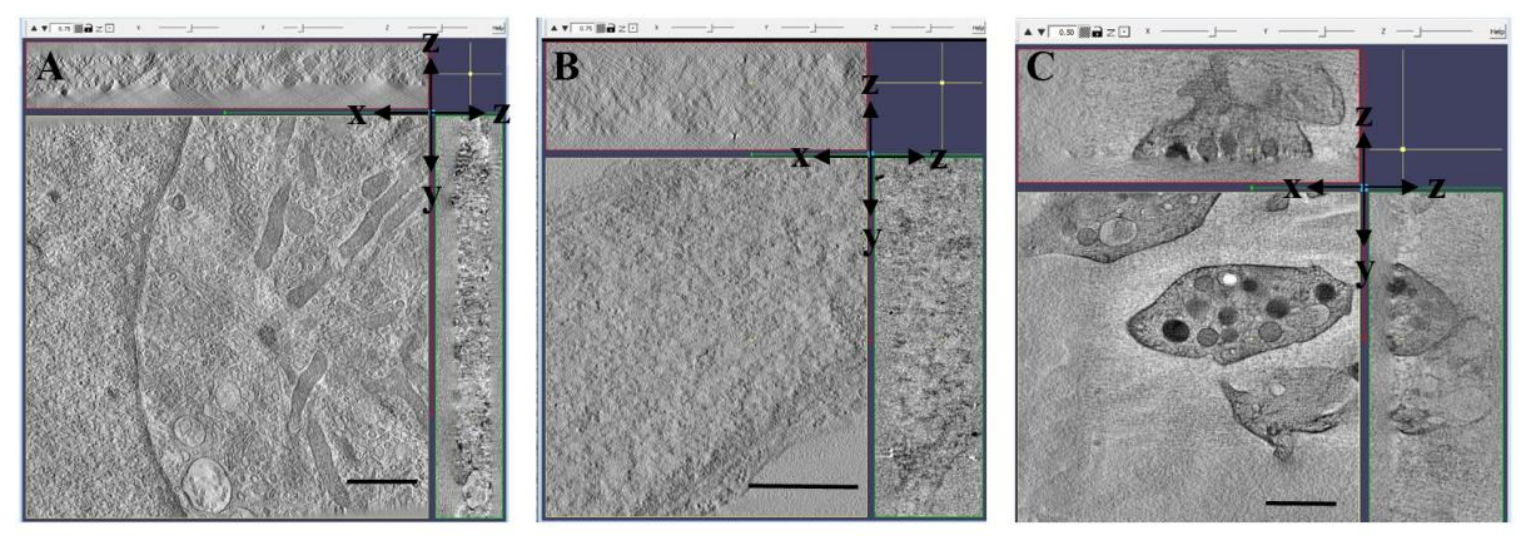

Figure 1. Usable specimen thickness in bright-field STEM tomograms of thick sections of stained cells, acquired at $300 \mathrm{keV}$ beam energy. (A) Orthoslices through a relatively heavily stained section of retinal pigment cells, shows that it is feasible to obtain high quality $3 \mathrm{D}$ reconstructions from a specimen of thickness of $500 \mathrm{~nm}$. (B) Orthoslices of thicker section of retinal pigment cells, shows that the quality of 
3D reconstructions deteriorates at a thickness of $750 \mathrm{~nm}$. (C) Orthoslices through a relatively lightly stained section of human blood platelets cell shows that it is feasible to obtain high quality 3D reconstructions from a specimen of thickness of $\sim 1.4 \mu \mathrm{m}$. Scale bar, $1 \mu \mathrm{m}$.

References

[1] A. Fera, Q. He, G. Zhang, R.D. Leapman, J. Microscopy. https://doi.org/10.1111/jmi.12865

[2] A.A. Sousa et al, Ultramicroscopy 109 (2009) p. 213.

[3] A.A. Sousa et al, J. Struct. Biol. 174 (2011) p. 107.

[4] This research was supported by the intramural program of the National Institute of Biomedical Imaging and Bioengineering, National Institutes of Health. 